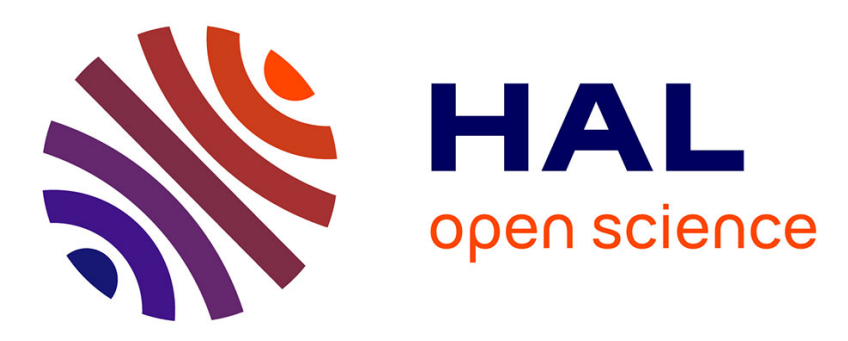

\title{
A new method to prepare microparticles based on an Aqueous Two-Phase system (ATPS), without organic solvents
}

Florence Dumas, Jean-Pierre Benoit, Patrick Saulnier, Emilie Roger

\section{- To cite this version:}

Florence Dumas, Jean-Pierre Benoit, Patrick Saulnier, Emilie Roger. A new method to prepare microparticles based on an Aqueous Two-Phase system (ATPS), without organic solvents. Journal of Colloid and Interface Science, 2021, 599, pp.642-649. 10.1016/j.jcis.2021.03.141 . hal-03388118

\section{HAL Id: hal-03388118 \\ https://univ-angers.hal.science/hal-03388118}

Submitted on 23 Nov 2021

HAL is a multi-disciplinary open access archive for the deposit and dissemination of scientific research documents, whether they are published or not. The documents may come from teaching and research institutions in France or abroad, or from public or private research centers.
L'archive ouverte pluridisciplinaire HAL, est destinée au dépôt et à la diffusion de documents scientifiques de niveau recherche, publiés ou non, émanant des établissements d'enseignement et de recherche français ou étrangers, des laboratoires publics ou privés. 
A new method to prepare microparticles based on an Aqueous Two Phase system (ATPS), without organic solvents

Florence Dumas, Jean-Pierre Benoit, Patrick Saulnier, Emilie Roger

Micro et Nanomédecines translationnelles, MINT, UNIV Angers, UMR INSERM 1066, UMR CNRS 6021, Angers, France

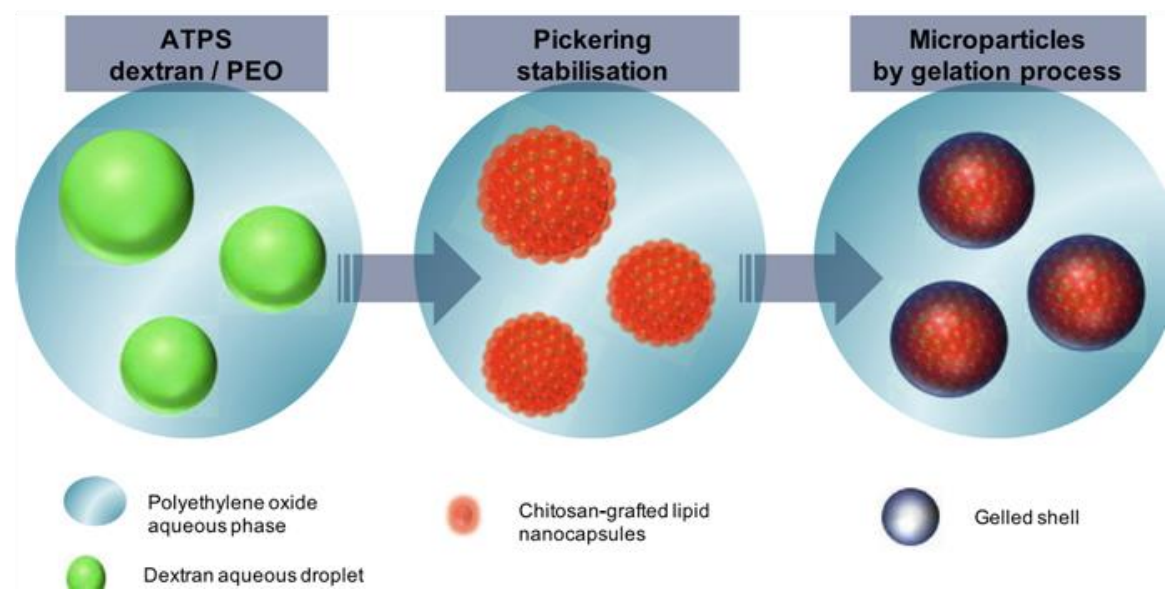

https://doi.org/10.1016/j.jcis.2021.03.141

Published in Journal of Colloid and Interface Science 599 (2021) 642-649 


\begin{abstract}
:
Hypothesis: Aqueous Two-Phase Systems (ATPS) are aqueous droplets dispersed in an aqueous phase. This specific behavior : least two water-soluble entities, such as thermodynamically incompatible polymers. A simple, fast, and "green" process to proc would be of high interest to the pharmaceutical field for drug delivery. However, to date, rapid destabilization of ATPS represe Herein we present a novel process to achieve a stabilized microparticle-ATPS, without the use of organic solvents.

Experiments: ATPS composed of dextran and polyethylene oxide were prepared. A Pickering-like emulsion technique was used to stabilize the ATPS by adsorbing semi-solid particles (chitosan-grafted lipid nanocapsules) at the interface between the two aqueous phases. Finally, microparticles were formed by a polyelectrolyte complexation and gelation. The structure and stability of ATPS were characterized using microscopy and Turbiscan analysis.
\end{abstract}

\section{Introduction}

Aqueous Two-Phase Systems (ATPS) are formed by combining a pair of incompatible hydrophilic polymers, or by combining a polymer and salt in water, leading to a partitioning of each polymer (or salt) in each one of the aqueous phases [1]. From their historical discovery by Martinus Beijerinck in 1896, ATPS have been employed for many applications [2,3]. Due to their biodegradability [4,5], polyethylene glycol (PEG) and dextran are commonly used for ATPS formation [2]. PEGdextran ATPS are mainly used for the purification or isolation of biomolecules such as proteins including monoclonal antibodies [2,6] (like IgG [7]) and enzymes (like dehydrogenase formate [8]). In some cases, ATPS have been optimized by chemical modification of PEG and/or dextran for recovery and purification of bioproducts leading to aqueous twophase affinity partitioning (ATPAP) [9]. Other applications using PEG-dextran ATPS include cell micropatterning techniques, biochemical analysis such as ATPS-ELISA or Alphalisa [7], blood cell separation [2], microfluidic applications [7,10], and extractive fermentation to optimize production yields of bioproducts [2]. Additionally, dextran microspheres have been produced from PEG and methacrylated dextran ATPS via a polymerization process [11], and more recently, microcapsules were formulated from PEGdextran ATPS by interfacial complexation [12]. The two latter systems were developed for biomedical applications such as encapsulation of cells or bacteria.

Despite their widespread use, understanding the partition process involved in the formation of an ATPS remains a challenge [2,6]. Phase diagrams of ATPS formulated from dextran and PEO (a high molecular weight PEG) have been described in the literature, and the concentration conditions for ATPS formation have been determined [13,14]. The importance of using purified PEO (without silica impurities) has been highlighted, and the interest of using polymers in powder-form to allow a simple one-step process for ATPS formulation was demonstrated [13].

Nevertheless, the main challenge with ATPS formulations remains their stabilization [10]. Indeed, ATPS are a complex dynamic system, and their structure as well as their kinetics of phase separation depend on the balance between viscous and interfacial forces [15]. Thus, it was well-known that the phase separation between PEO and dextran is affected by temperature, by changing the polymer concentration and changing the molar mass of one of the polymers [16]. Thus kinetic stabilization is required via adsorption of stabilizing species (copolymers [17] or particles) at the liquid-liquid interface or via interfacial complexation [10]. The strategy which consists of adsorbing solid particles at the interfaces of emulsion phases is called the Pickering method [18] and different kinds of particles such as silica [19], latex [20], or carboxylated particles [21], as well as with proteins such as blactoglobulin [22], whey protein [23] and zein-based nanoparticles [24] have already been studied to stabilize ATPS. In doing, among new perspectives in the area of ATPS, preparing biocompatible microparticles with a great potential in biomedical application is a current challenge [10]. In this goal, here we describe how to formulate stable microparticles from an ATPS based on PEO (molecular weight $200 \mathrm{kgmol}^{1}$ ) and dextran (molecular weight $500 \mathrm{kgmol}^{1}$ ) mixtures. In order to stabilize this system, a Pickering-like technique was used with lipid nanocapsules (LNCs).

LNCs are non-solid particles, biocompatible, prepared via a solvent free process, with film-forming properties [25,26]. Their formulation and composition lead to $20-100 \mathrm{~nm}$ diameter nanocapsules with an oily core and a surfactant shell [27]. The structure and stability of these novel microparticles was studied, and their potential interest in pharmaceutical applications is discussed.

\section{Materials and methods \\ 2.1. Materials}

Chitosan oligosaccharide lactate 5,000 Da, polyethylene oxide (PEO) (Mw: $200 \mathrm{kgmol}^{1}$ ), alginic acid sodium salt from brown algae (low viscosity), calcium chloride dihydrate, dextran and fluorescein isothiocyanate (FITC) dextran (Mw: $500 \mathrm{kgmol}^{1}$ ) from Leuconostoc spp. were provided by SigmaAldrich (Saint-Quentin Fallavier, France). Labrafac WL 1349 (caprylic-capric acid triglycerides) was kindly provided by 
Gattefossé S.A. (Saint-Priest, France). LipoïdS75-3 (soybean lecithin at $69 \%$ of phosphatidylcholine) was provided by Lipoïd Gmbh (Ludwigshafen, Germany). Kolliphor HS 15 (mixture of free polyethylene glycol 660 and polyethylene glycol 660 hydroxystearate) was provided from BASF (Ludwigshafen, Germany). 1,10-dioctadecyl-3,3,30,30-tetramethylin dodicarbocyanine 4-chlorobenzenesulfonate (DiD) was provided by Molecular probes (Eugene, USA). MilliQ water obtained from a MilliQ filtration system (Millipore, Paris, France).

2.2. Methods

2.2.1. Formulation of LNCs labelled with DiD dye

The formulation of $50 \mathrm{~nm}$ diameter LNCs was based on the phase inversion process defined by Heurtault et al. [28]. Briefly, lipophilic fluorescent dye $\mathrm{DiD}$ was first dissolved in LabrafacWL1349 (at $3 \mathrm{mgg}^{1}$ wt ratio dye/Labrafac). Then, DiDLabrafac, LipoïdS75-3, KolliphorHS15, NaCl and water $(800,67,600,75$ and $2300 \mathrm{mg}$, respectively) were mixed under magnetic stirring. The mixture was heated up to $90 \mathrm{C}$ and then cooled down to $60 \mathrm{C}$, repeatedly for three cycles. At the end of the last cycle, the formulation was rapidly diluted with $5 \mathrm{~mL}$ of water at $4 \mathrm{C}$, to create a suspension of LNCs. The LNC suspension was then filtered through a $0.22 \mathrm{~mm}$ Clearline filter with PES membrane (Biosigma S.r.l., Cona, Italy).

\subsubsection{Formulation of chitosan-grafted LNCs (LNCs-CS)}

Chitosan was grafted onto the terminal hydroxy group of KolliphorHS15 at the surface of the LNCs by a transacylation process, as described in the literature [29]. Briefly, chitosan was dissolved in $\mathrm{NaOH} 10 \mathrm{M}$, then the LNCs were added in order to obtain a final concentration of chitosan of $1.2 \mathrm{mgmL}^{1}$ and $5 \%$ $(\mathrm{v} / \mathrm{v})$ for $\mathrm{NaOH}$. The mixture was left to react under magnetic stirring for $15 \mathrm{~min}$ at $25 \mathrm{C}$. The transacylation reaction was then stopped by adding glycine buffer $0.05 \mathrm{M} / \mathrm{pH} 2.2$ with a ratio buffer:LNCs of 1:1 (v/v). Then, the LNCs-CS suspension was purified by dialysis against water for $24 \mathrm{~h}$ with a $100 \mathrm{kDa}$ molecular weight cut-off point dialysis membrane (provided by Spectrum Laboratories, Rancho Dominguez, USA), to remove free chitosan. The LNCs-CS suspension was finally filtered $(0.22 \mathrm{~mm})$.

\subsubsection{LNCs characterization}

Suspensions of LNCs and LNCs-CS were characterized by dynamic light scattering (DLS) analysis on a Zetasizer Nano series (Malvern Instruments S.A., Worcestershire, UK), to determine their size distribution, polydispersity index (PDI) and their charge. Each measurement was run in triplicate. Suspensions of LNCs and LNCsCS were diluted at 1:60 (v/v) and $1: 30(\mathrm{v} / \mathrm{v})$ in water, respectively, prior to the analyses. The particle concentrations were determined by nanoparticle tracking analysis (NTA) using a NS300 Nanosight (Malvern Instrument, Worcestershire, UK) with a low-volume flow cell plate and a $405 \mathrm{~nm}$ laser. The nanoparticle suspensions were injected directly into the chamber using a $1 \mathrm{~mL}$ syringe installed on a syringe pump. NTA analytical software (version 3.2), was used to analyze video images. The measurements were made at room temperature, and each video sequence was captured over $60 \mathrm{~s}$. Each analysis was repeated five times for each sample. Concentrations inferior to 5 particles/frame were considered as being unreliable, and the corresponding samples were considered as having no particles. LNCs and LNCs-CS suspensions were diluted in ultrapure water by a factor of 1000 000 or $500000(\mathrm{v} / \mathrm{v})$ prior to analysis.

\subsubsection{PEO purification process}

Impurities contained in the PEO were removed by centrifuging the polymer dissolved in water (centrifuge 5810R, Eppendorf AG Hamburg, Germany) for 5 min at 4000 rpm, 3 $220 \mathrm{~g}$. The supernatant was separated from the impurities pellet, and then centrifuged under the same conditions until no pellet was formed. To obtain a purified PEO powder, the supernatant was then freeze-dried (Lyovac GT2 (Steris, Germany)/CC-505 (Huber, Germany)).

\subsubsection{Preparation of ATPS}

Dextran and purified PEO powders were weighed and mixed. Labelled dextran, water and, for some preparations LNCs (LNCs or LNCs-CS), were then added, and the mixture was stirred until complete dissolution of polymers. Systems were mixed under magnetic stirring for $15 \mathrm{~min}$. The final concentrations are reported in Table 1.

\subsubsection{Preparation of microparticles}

ATPS were prepared as described above, but the stirring step was performed in an ice-bath. Then, alginate solution at 10 $\mathrm{mgmL}^{1}$ and of calcium chloride solution at $10 \mathrm{mgmL}^{1}$ were added to ATPS (concentrations are reported Table 1). The stirring was maintained (still in an ice-bath) for $30 \mathrm{~min}$ after each addition, respectively. Finally, stirring was stopped, and the formulation was left to gel at $4 \mathrm{C}$.

\subsubsection{Characterization of ATPS}

ATPS contained in $25 \mathrm{~mL}$ microarray slides, were analyzed by confocal microscopy (Leica TCS SP8 laser-scanning confocal micro- scope, Leica Microsystems, Heidelberg, Germany) with a x63 PL APO (N.A. 1.4) objective and 3 zoom. Excitation wavelengths were set at $488 \mathrm{~nm}, 561 \mathrm{~nm}$ and $633 \mathrm{~nm}$ respectively for FITC, TRITC and DiD, and fluorescence was collected between $491-565 \mathrm{~nm}, 564-618 \mathrm{~nm}$ and 636-686 nm for each dye, respectively. 
2.2.8. Characterization of microparticles

Gelled ATPS were characterized using confocal microscopy and by Transmission Electron Microscopy (TEM, JEOL JEM1400, JEOL, Croissy-sur-Seine, France).

\subsubsection{Stability studies of ATPS and microparticles}

Stability assays were performed on ATPS (without LNCs, and with LNCs or LNCs-CS) and on microparticles using two methods: Multiple Light Scattering (Turbiscan measurement) and confocal microscopy.

Using a Turbiscan TOWER (Formulaction, Toulouse, France), samples were analyzed in $4 \mathrm{~mL}$ cells at $4 \mathrm{C}$, over 28 days, according to the following schedule: 1 scan every $10 \mathrm{~min}$ for the 12 first hours, then 1 scan every 15 min for the next 36 h, then 1 scan every 35 min for 6 days and finally, 1 scan at day 14, 21 and 28. For each sample analyzed, the Turbiscan Stability Index (TSI) was determined by the Turbisoft software. TSI values were calculated at time $\mathrm{t}$ from transmission and backscattering data according to equation (1).

$$
\operatorname{TSI}(t)=\frac{1}{N_{h}} \sum_{t_{i}=1}^{t_{\max }} \sum_{z_{i}=z_{\min }}^{z_{\max }}\left|B S T\left(t_{i}, z_{i}\right)-B S T\left(t_{i-1}, z_{i}\right)\right|
$$

where $t_{\max }$ is the measurement point corresponding to the time $t$ at which the TSI is calculated, $z_{\min }$ and $z_{\max }$ the lower and upper selected height limits respectively, $\mathrm{N}_{\mathrm{h}}=\left(\begin{array}{ll}\mathrm{Z}_{\max } & \mathrm{Z}_{\min }\end{array}\right) / \mathrm{Dh}$ the number of height positions in the selected zone of the scan and BST the signal used (backscattering if transmission $<0.2 \%$, or transmission otherwise).

All of the raw profiles obtained via Turbiscan analysis (transmission and backscattering signals) are available in the Supporting Information (Figs. S1, S2, S3 and S4).

With confocal microscopy, samples were analyzed over 28 days (at 0, 7, 14 and 28 days). They were also analyzed after dilution in water (at 1:2 (v/v) ratio) and after centrifugation (at $10,000 \mathrm{rpm}, 9610 \mathrm{~g}$ for $15 \mathrm{~min}$ ) at day 0 . After centrifugation, the supernatant was discarded, and the pellet was resuspended in the same volume of water, prior to imaging.

\section{Results and discussion}

\subsection{ATPS formulation}

\subsubsection{ATPS characterization}

ATPS are usually characterized with phase diagrams, which express the concentration of one polymer as a function of the other, and show the binodal lines separating ATPS and no ATPS areas, as well as the tie-lines [30]. Based on our previous work, a dextran/PEO ATPS was formulated from polymers in powder form

with purified PEO [13]. Fig. 1 shows the phase diagram for this system, and the formulation concentrations studied herein $(0.5 \%(\mathrm{w} / \mathrm{v})$ of dextran and $4.0 \%(\mathrm{w} / \mathrm{v})$ of PEO) are pinpointed, along with a confocal microscope image of the ATPS formed with this composition. According to the phase diagram (at 25 C), and as confirmed by the confocal microscopy analysis, the ATPS was composed of a dextran discontinuous phase (dextran droplets, in green) and a PEO continuous phase (unlabeled).

\subsubsection{ATPS stability}

The polymer concentrations used in this study were relatively close to the binodal line of the phase diagram, where the system is the least stable. Consequently, it was important to find methods to properly characterize the stability of this ATPS.

Dilution in pure water [31] or applying a centrifugal force are two methods that are widely used to evaluate the physical stability of emulsions. Therefore, the physical stability of our ATPS was studied after dilution and after centrifugation, and we also used confocal microscopy imaging over 28 days to assess the stability over time (see Fig. 2). After centrifugation of the non-stabilized ATPS, a phase separation occurred: the FTIC-dextran was in the bottom phase, and no ATPS structures could be observed by confocal microscopy (Fig. 2-1F). After dilution, no dextran droplets could be observed (Fig. 2-1E) meaning that the ATPS had crossed the binodal line, resulting in phase separation and complete destabilization of the dextran droplets. In addition, the confocal microscopy time-stability study revealed a significant coalescence phenomenon which affected the dextran droplets from day 7 onwards (Fig. 2-1B, C and D). This result was also confirmed with the Turbiscan TOWER analysis. The stability results for the non-stabilized ATPS (transmission profile) are shown in Fig. 3 (orange line). A TSI value of 10 was reached before $12 \mathrm{~h}$ of analysis, and the

Table 1: Composition of formulations. (ATPS: Aqueous Two-Phase System, LNCs: lipid nanocapsules, CS: chitosan, PEO: polyethylene oxide, FITC: fluorescein isothiocyanate).

Table 1: Composition of formulations. (ATPS: Aqueous Two-Phase System, LNCs: lipid nanocapsules, CS: chitosan, PEO: polyethylene oxide, FITC: fluorescein isothiocyanate).
$\begin{array}{llllll}\text { Dextran } & \text { PEO } & \text { LNCs suspension } & \text { LNCs-CS suspension } & \text { Alginate solution } \\ {[\mathrm{wt} \%]} & {[\mathrm{wt}} & {\left[\mathrm{particlesmL}^{1}\right]} & {\left[\mathrm{particlesmL}^{1}\right]} & {\left[\mathrm{mgmL}^{1}\right]}\end{array} \quad \begin{array}{llll}\text { Calcium chloride solution } \\ {\left[\mathrm{mgmL}^{1}\right]}\end{array}$

$\%]$

ATPS

ATPS with LNCs

ATPS with LNCs-CS

Gelled ATPS-LNCs-CS Microparticles
$0.5 \%$
(including $4.2 \%$ FITC-labelled) 
TSI value strongly increased with time, to reach a value over 55 , which demonstrates the rapid and complete destabilization of this nonstabilized ATPS.

\subsection{ATPS stabilization by a Pickering-like technique}

The Pickering technique consists of using solid particles, such latex or proteins, to stabilize the interface of emulsion droplets [14,22,32]. In contrast, herein we explored the potential of lipid nanocapsules (LNCs) for stabilizing ATPS. Because LNCs are considered as semi-solid particles resembling a hybrid of polymer nanoparticles and liposomes [33], we named it Pickering-like technique. LNCs, developed by our group, were chosen for this purpose because of their film-layer properties [25,26,34]. Moreover, due to the interesting properties of chitosan (CS) which provides network stabilization of emulsions by electrostatic interactions [35], we chose to graft chitosan onto the surface of the LNCs. These chitosan-grafted LNCs were called LNCs-CS. The impact of including the LNCs or LNCs-CS on the structure and stability of the ATPS was evaluated.

\subsubsection{LNCs characterization}

LNCs were formulated as described in the literature [28] and then characterized by dynamic light scattering (DLS) and nanoparticle tracking analysis (NTA) to determine their size (hydrodynamic diameter), polydispersity index (PDI), zeta potential and the concentration of particles. Table 2

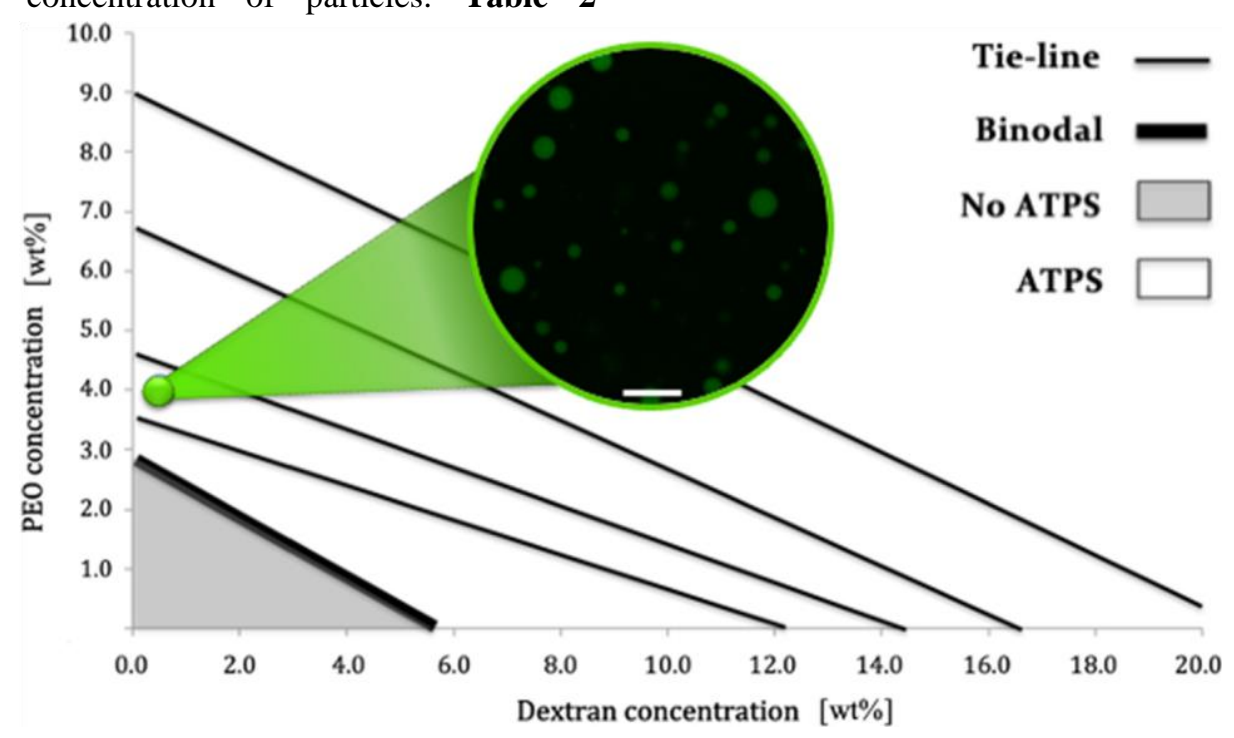

Fig. 1. Observation of the ATPS by confocal microscopy. FITC-labeled dextran droplets appear in green (scale bar: $10 \mathrm{~mm}$ ), and are located in a phase diagram (obtained from literature [13]) for dextran and purified PEO polymers in water via Turbiscan analysis. The green point represents the polymer concentrations used for our ATPS formulation. Adapted with permission from Dumas et al. [13] Copyright 2020, Colloid and Polymer Science. (ATPS: Aqueous Two-Phase System, PEO: polyethylene oxide). summarizes the characteristics of the LNCs formulations. LNCs had a size close to $50 \mathrm{~nm}$, with a slightly negative zeta potential, and a PDI of 0.06 indicating a narrow distribution of particles. These characteristics are in consistence with other publications, indeed, LNCs are widely described and fully characterized in the literature (DLS, AFM, TEM) [28,36,37]. Grafting chitosan onto the LNCs slightly increased the hydrodynamic diameter as well as the polydispersity. Nevertheless, the PDI was still below 0.2 demonstrating the monodispersity of the suspension. The particle concentration decreased after adding chitosan due to the dialysis, which diluted the samples. The zeta potential was close to neutral for LNCs (4.1 mV) and surprisingly, negative for LNCs-CS (21.9 $\mathrm{mV}$ ). Indeed, we had expected to form positively charged LNCs-CS by grafting positively charged CS onto the LNCs surface. In the literature [29], for the same conditions and CS concentration, the zeta potential was found to be $+24.7 \pm 2.1$ $\mathrm{mV}$. One explanation to explain the negative charge of the LNCs-CS could be due to the highly basic conditions $(\mathrm{NaOH}$, $10 \mathrm{M}$ ) used in transacylation reaction, compared to the milder conditions found in literature [38]. Thus, to confirm this hypothesis, we formulated LNCs using the same reaction conditions $(\mathrm{NaOH}, 10 \mathrm{M})$, without grafting chitosan onto the LNCs, and we found that the LNCs had a negative global charge $(21.6 \mathrm{mV}, \mathrm{n}=1)$ (results not presented). So, the negative charge was due to the conditions and grafted chitosan did not modified it. 

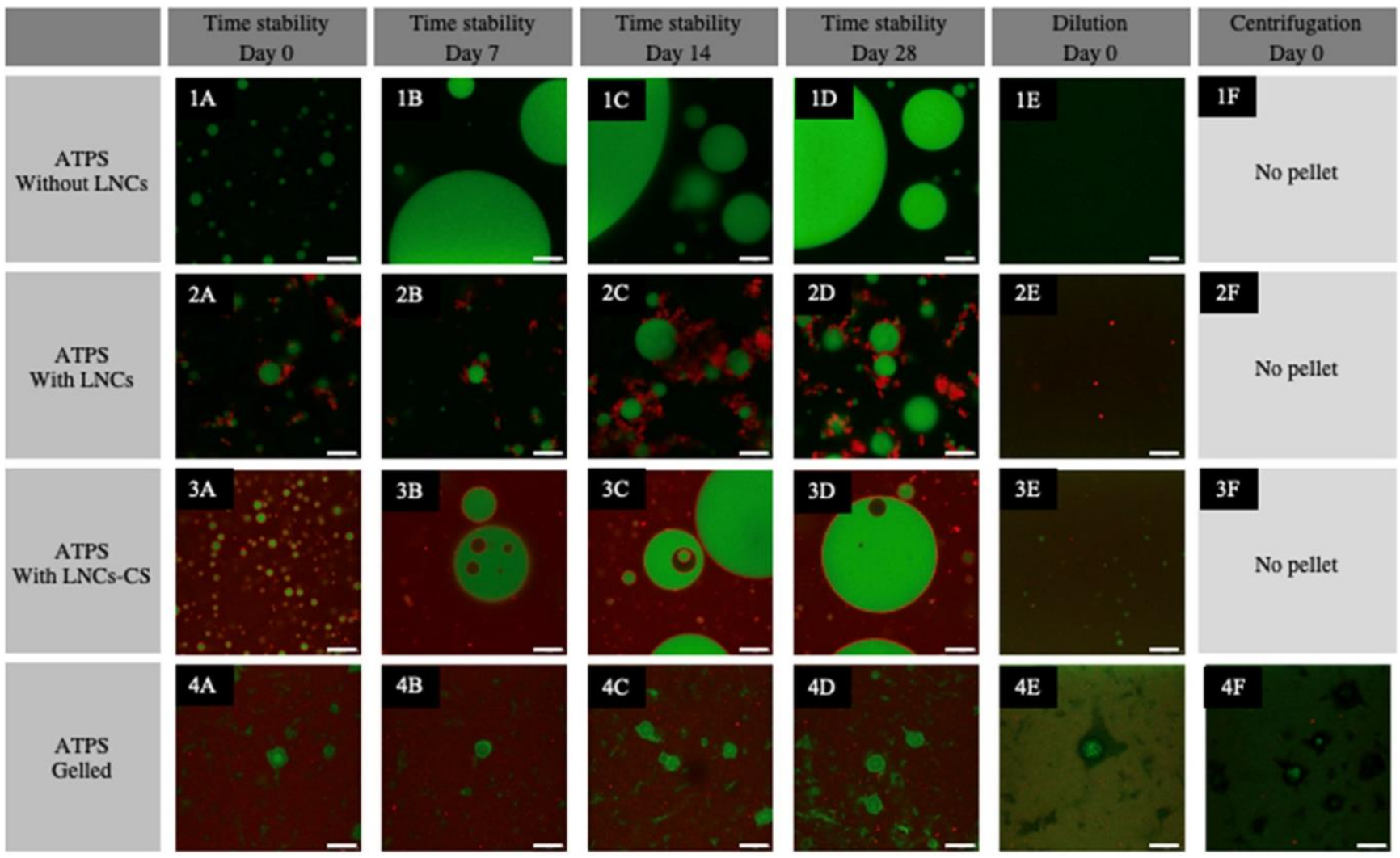

Fig. 2. ATPS without LNCs (1), with LNCs (2), with LNCs-CS (3) and gelled LNCs-CS (4): at day 0 (A), day 7 (B), day 14 (C) and day $28(D)$, as well as, stability against dilution in water $(E)$ and centrifugation $(F)$. Observations via confocal microscopy. FITClabeled dextran appears in green and DiD-labeled LNCs appear in red. Scale bar: 10 mm. (ATPS: Aqueous Two-Phase System, LNCs: lipid nanocapsules, CS: chitosan).

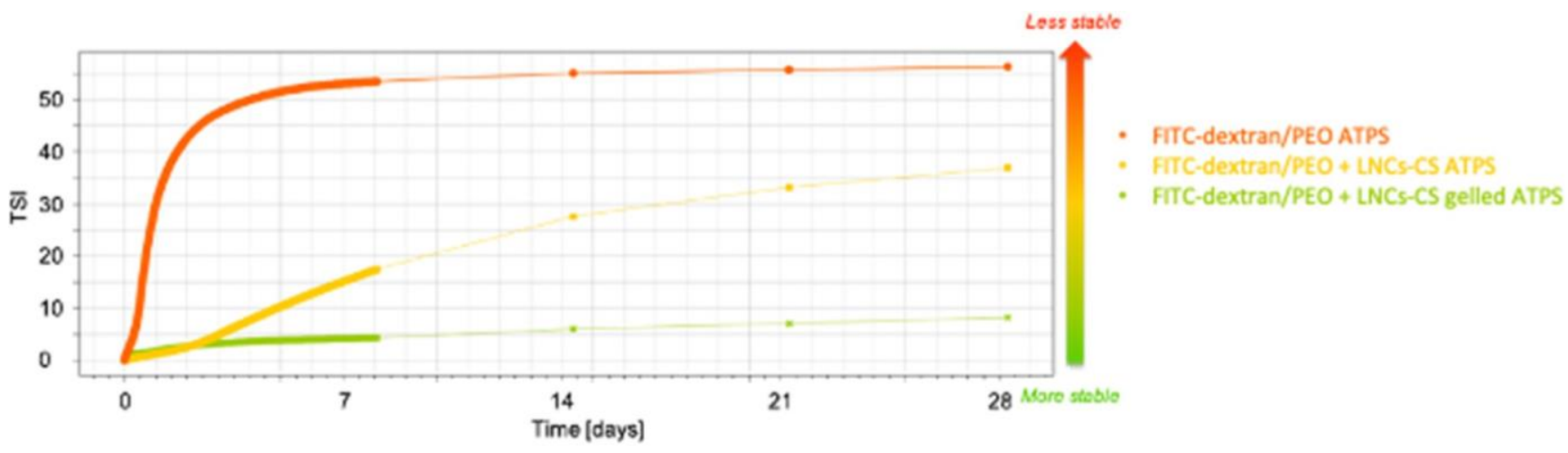

Fig. 3. Turbiscan Stability Index (TSI) comparison of 3 ATPS systems at 4 C, obtained from transmission profiles over 28 days, for i) FITC-dextran/PEO ATPS (in orange), ii) FITC-dextran/PEO + LNCs-CS ATPS (in yellow) and iii) FITC-dextran/PEO + LNCsCS gelled ATPS (in green). (For interpretation of the references to color in this figure legend, the reader is referred to the web version of this article.) 
Table 2

Characterisation of LNCs (lipid nanocapsules) and LNCs-CS (lipid nanocapsules chitosan) suspensions. Size, PDI (polydispersity index), zeta potential and concentration were obtained from 6 measurements (in triplicate each).

\begin{tabular}{lll}
\hline & LNCs & LNCs-CS \\
\hline Size $[\mathrm{nm}]$ & $56.8 \pm 1.8$ & $63.4 \pm 1.3$ \\
PDI & $0.061 \pm 0.018$ & $0.133 \pm$ \\
& & 0.023 \\
Zeta Potential [mV] & $4.1 \pm 0.5$ & $21.9 \pm 3.5$ \\
Concentration [10 & & \\
particlesmL & &
\end{tabular}

of polyethylene glycol hydroxystearate (present in Kolliphor HS 15) during the transacylation process could explain this observation. Based on these results, it was assumed that the transacylation process led to a negative global charge on the LNCs even if chitosan was grafted onto LNCs.

\subsubsection{ATPS with LNCs formulation}

LNCs or LNCs-CS were added to the ATPS and their impact on the stability of the system was studied. The ATPS were imaged by confocal microscopy.

LNCs and LNCs-CS were observed surrounding dextran droplets, in clusters (Fig. 2-2A) or uniformly spread out (Fig. 2-3A). In both cases, this behavior demonstrated the clear-cut affinity of the LNCs and LNCs-CS for the interfaces between PEO and dextran. Similar coating behaviors have been observed with the Pickering technique when using solid particles such as latex, which become trapped at the interface of the dextran droplet in the PEO continuous aqueous phase [14]. A film-layer was formed only by the LNCsCS (Fig. 2-3A). So, even if chitosan grafted onto LNCs was not demonstrated by zeta potential values, in the presence of PEO/Dextran, the formation of a chitosan network between LNCs-CS at the interface of dextran droplets can be assumed. In the literature, blactoglobulin particles [22] as well as latex particles [14] were also trapped at the interface of dextran droplets.

\subsubsection{Stability of ATPS with LNCs}

The stability of the ATPS with LNCs and LNCs-CS was then evaluated. Applying a centrifugal force (10,000 rpm, $9610 \mathrm{~g}$ for $15 \mathrm{~min}$ ) to the ATPS with LNCs and LNCs-CS did not form any pellet (Fig. 2). After dilution, no dextran droplet was observed for ATPS with LNCs (Fig. 2-2E). Thus, ATPS-LNCs (i.e. without chitosan) were easily destabilized. In contrast, after dilution of ATPS with LNCs-CS, small dextran droplets were observed (Fig. 2-3E). Pickering-like stabilization of the ATPS by the LNCs-CS was obtained. Coating with LNCs-CS helped to retain the dextran inside droplets under conditions where
ATPS alone, or ATPS-LNCs would be destabilized, and pass under the binodal line (Fig. 1).

The stability over 28 days was also studied for the ATPS formulated with LNCs and LNCs-CS. For the ATPS with LNCs (Fig. 2-2C) an increase in droplet size was observed from day 14 (compared to day 7 for ATPS without LNCs - Fig. 2-1B). In contrast, for ATPS with LNCs-CS, coalescence was observed from day 7 (Fig. 2-3B). Thus, grafting chitosan onto the LNCs did not improve the system stability. There are similarities between LNCs-ATPS system and that studied by Balakrishnan and Nguyen works who studied the effect of adding latex particles and protein particles [14,22] to mixtures of aqueous dextran and PEO solutions. Indeed, in the presence blactoglobulin particles, a complete sedimentation of dextran droplet was observed after 1 week even if b-lactoglobulin particles covered the droplet [22]. Similarly, latex particles did not have a significant stabilizing effect.

Interestingly, after adding LNCs-CS onto ATPS, the formation of multiple emulsions such as $\mathrm{PEO} /$ dextran/PEO and even dextran/ PEO/dextran/PEO (Fig. 2-3B, C and D) was observed from day 7 onwards. The formation of multiple emulsions from ATPS composed of dextran (containing a positively charged polyelectrolyte) and PEG, has been reported previously in the presence of $\mathrm{SiO}_{2}$ nanoparticles [39]. In this publication, authors propose a mechanism behind the formation of double emulsion linked to the concentration of nanoparticles in the PEG phase. Indeed, a rapid membrane was formed via interfacial complexation of polyelectrolyte and $\mathrm{SiO}_{2}$ nanoparticles. Due to the concentration of nanoparticles in the PEG phase, some nanoparticles were excluded from the dextran phase, and an osmotic stress imbalance was created. Then, water from the dextran phase was removed and droplets of PEG phase were drawn into the dextran droplets to form the double emulsion interior [39].

Stability assays of our ATPS-LNCs were also performed by Turbiscan analysis. The profile corresponding to ATPS with LNCs was obtained with a backscattering signal due to the opacity of the sample analyzed. Because it was not accurate to compare TSI obtained from transmission and backscattering profiles, the TSI graph for ATPS with LNCs is not presented here. The results of the transmission and backscattering profiles obtained for ATPS with LNCs (Fig. S2) were consistent with the relative stability observed under confocal microscopy (Fig. 2-2). The result obtained for ATPS with LNCs-CS is shown in Fig. 3 (yellow line). Fig. 3 shows that adding LNCs-CS to ATPS improved the stability, as compared to ATPS without LNCs (orange line), however, a slow destabilization was observed with a TSI value of 10 being reached before day 5 . The ATPS with LNCs and LNCs-CS were clearly not sufficiently stable for use as a drug carrier system. 


\subsection{Microparticles}

\subsubsection{Microparticle formulation}

To consolidate the LNCs' coating, the formulation process was optimized. Hann et al. (2017), formed a rigid shell by complexation of polyelectrolytes at the outer interface between a PEG phase with negatively charged $\mathrm{SiO} 2$ nanoparticles and a dextran phase with positively charged polyelectrolytes [39]. In order to improve the stability of the ATPS with LNCs-CS, the well-defined polyelectrolyte complexation properties of chitosan $[40,41]$ were used, by coupling it with alginate to form a gel. Fig. 2-4A shows the impact of the gelation process on the ATPS. Thick shells were observed surrounding the dextran droplets. The green color observed (irrespective of the focal plane) was not due to alginate autofluorescence, and thus it was assumed to indicate dextran-FITC escape from the core.

\subsubsection{Stability of microparticles}

The stability of the gelled system was evaluated. After centrifugation, the gelled ATPS-LNCs-CS produced a pellet. The supernatant was removed and replaced by water to obtain the same concentration without dilution. This preparation was then analyzed by confocal microscopy, and dextran droplets were observed (Fig.2-4F).After dilution, dextran droplets were still observed (Fig. 2-4E). In contrast with previous ATPS formulations, submitting gelled ATPS-LNCs-CS to dilution in

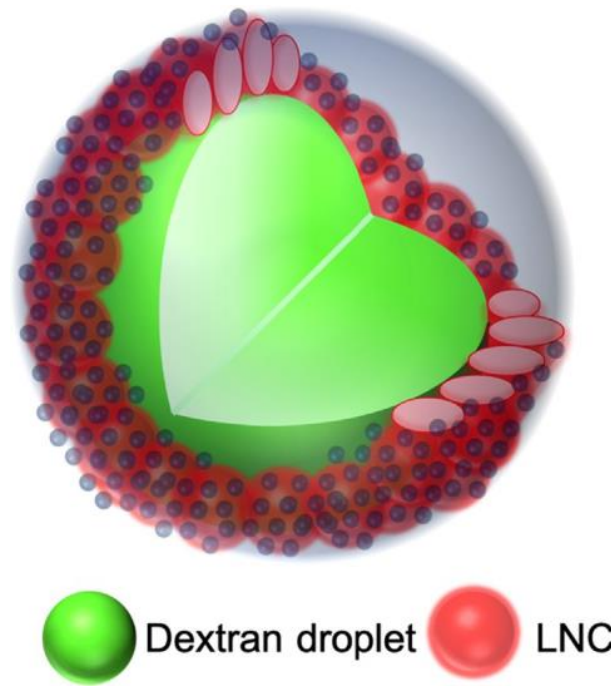

(A)

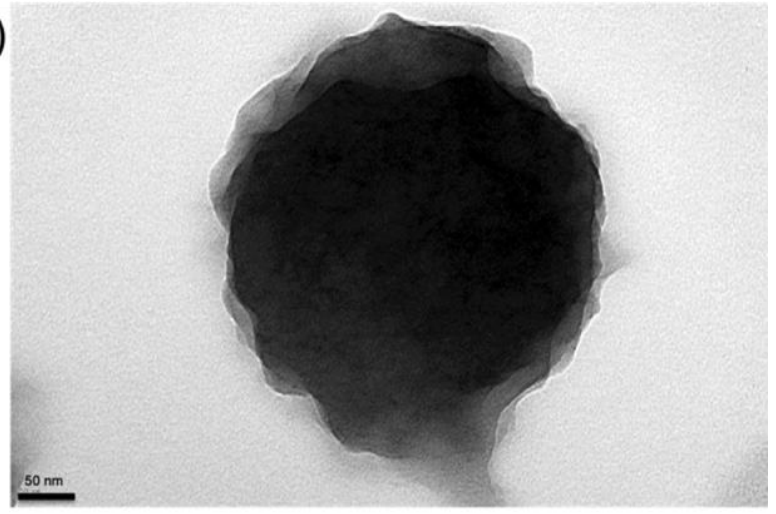

(B)

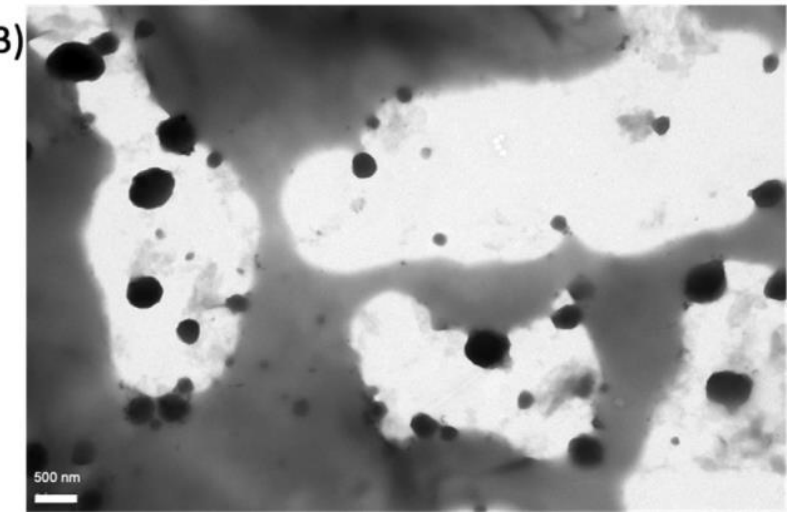

Fig. 4. Transmission Electron Microscopy micrograph of gelled ATPS-LNCs-CS. (A) Scale bar: $50 \mathrm{~nm}$, (B) Scale bar: $500 \mathrm{~nm}$.

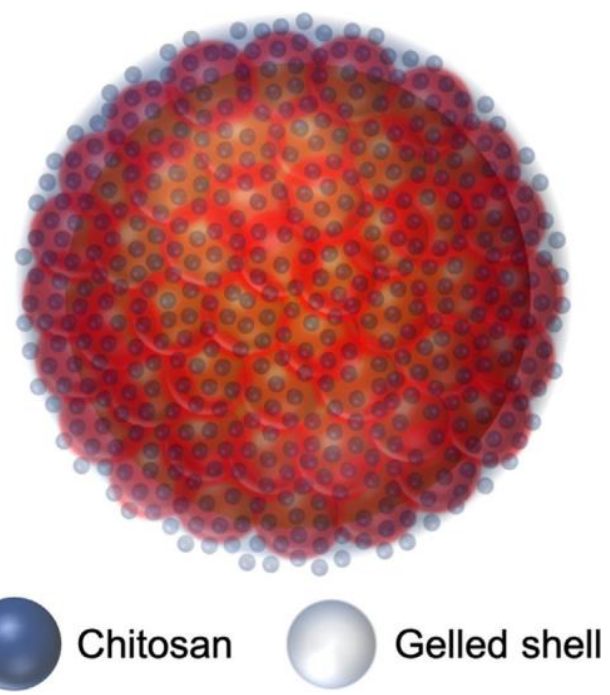

Fig. 5. Schematic representation of the gelled ATPS-LNCs-CS microparticles with chitosan (in blue) grafted onto the lipid nanocapsules (LNCs) (in red), which surround dextran droplets (in green). The grey shroud represents an alginate gelled shell attached to the chitosan by a gelation process with calcium chloride.

water did not impact their structure. This highlights the interest of strengthening the LNC-CS film-layer by an alginate gelation process for applications in diluted conditions. Finally, the stability over time was studied for the gelled ATPS-LNCs-CS formulation.
With confocal microscopy, no sign of destabilization was observed until day 28 for the gelled ATPS-LNCs-CS (see Fig. 2-4B and C). At day 28, a few dextran droplet clusters were observed but no coalescence phenomenon was noticed (Fig. 24D). Moreover, by Turbiscan TOWER analysis, a good 
stability profile was obtained for 28 days, during which the TSI value remained below 10 (Fig. 3, green line). These results demonstrate that the gelled ATPS-LNC-CS are stable for at least 28 days in solution at $4 \mathrm{C}$.

\subsubsection{Microparticle characterization}

Due to their good stability, Transmission Electron Microscopy (TEM) analysis of the gelled ATPS-LNCs-CS formulation was performed after 2 days of gelation. Spherical structures inferior at $10 \mathrm{~mm}$ were observed (Fig. 4). The shape of the structures was consistent with the dextran droplets observed in the gelled ATPSLNCs-CS formulation using confocal microscopy (Fig. 2-4A). The irregular surface of the microparticles observed in Fig. 4 is believed to be due to the LNCs-CS surrounding the dextran droplets. The proposed structure of the gelled ATPS-LNCs-CS microparticles is shown in Fig. 5. The rugged microparticle surface observed by TEM (Fig. 4) fits the proposed structure model (Fig. 5), with LNCsCS coating onto the surface of the dextran droplets, surrounded by a gelled chitosan-alginate network.

\section{Conclusion}

Due to their biocompatibility, and an easy, solvent-free formulation process, ATPS are already used for many applications, especially in the biomedical field. However, the poor stability of these classical ATPS systems remains a major obstacle for further development of ATPS, especially with respect to their use in the drug delivery field. This work demonstrated that stable microparticles with a size lower than $10 \mathrm{~mm}$ could be formed from an ATPS, via a gelation process based on a Pickering-like technique using chitosan grafted lipid nanocapsules (LNCs-CS). Most microparticle formulation processes involve the use of organic solvents, however, in contrast this novel gelled ATPS-LNCs-CS process avoids organic solvents and is thus of potential pharmaceutical use in the development of a microparticulate drug delivery system. The microparticles formulated herein were stable in solution at $4 \mathrm{C}$ for at least 28 days, and upon dilution and centrifugation. To date, only Ma et al. (2016), have developed microcapsules from an ATPS by polyelectrolyte complexation, and adjusted the shell thickness by adding polyelectrolyte layers [42]. However, they obtained substantially larger particles (above $100 \mathrm{~mm}$ ) and did not short-term stability. A potential pharmaceutical application of the microparticles developed in this study will be for oral drug administration. Indeed, microscale carriers have interesting properties for oral drug delivery such as taste-masking, sustained/targeted release, and in addition polycationic polymers can improve oral bioavailability [43]. For example, oral delivery of molecules in class III of the Biopharmaceutical Classification System (i.e., high solubility, low permeability), as well as oral delivery of peptides and proteins, still remains a technological challenge, which may be facilitated via the use of a gelled-ATPS-LNCs-CS formulation. Moreover, the surface of the microparticles could be further modified with other molecules (e.g., enteric coatings or targeting moieties) so as to allow targeted gastro-intestinal (GI) drug delivery.

Further work is required to characterize the gelled ATPSLNCsCS microparticles, including a detailed exploration of their structure, their encapsulation and (sustained/targeted) drug release capabilities, as well as their long-term stability (e.g., after freezedrying). Nevertheless, the formulation of stable, biocompatible microparticles from an ATPS via the innovative, solvent-free process detailed herein, constitutes the first step to a potentially large range of applications for these microparticles.

\section{Declaration of Competing Interest}

The authors declare that they have no known competing financial interests or personal relationships that could have appeared to influence the work reported in this paper.

\section{Acknowledgements}

We gratefully thank the SCIAM (Service Commun d'Imageries et d'Analyses Microscopiques; Université d'Angers), especially Rodolphe Perrot for confocal microscopy imaging and Romain Mallet for TEM imaging.

The authors also acknowledge the help of Dr. Paul Baldwin for preparation of the manuscript.

Supplementary data to this article can be found online at https://doi.org/10.1016/j.jcis.2021.03.141.

\section{References}

[1] R. Hatti-Kaul, Aqueous Two-Phase Systems: A General Overview, Mol. Biotechnol. 19 (2001) 269-278, https://doi.org/10.1385/MB:19:3:269.

[2] M. Iqbal, Y. Tao, S. Xie, Y. Zhu, D. Chen, X. Wang, L. Huang, D. Peng, A. Sattar, M. A.B. Shabbir, H.I. Hussain, S. Ahmed, Z. Yuan, Aqueous two-phase system (ATPS): an overview and advances in its applications, Biol. Proced. Online. 18 (2016) 18, https://doi.org/10.1186/s12575-016-0048-8

[3] J.A. Asenjo, B.A. Andrews, Aqueous two-phase systems for protein separation: Phase separation and applications, J. Chromatogr. A. 1238 (2012) 1-10, https:// doi.org/10.1016/j.chroma.2012.03.049.

[4] M.E. Ali, A. Lamprecht, Polyethylene glycol as an alternative polymer solvent for nanoparticle preparation, Int. J. Pharm. 456 (2013) 135-142, https://doi. org/10.1016/j.ijpharm.2013.07.077.

[5] A. George, P.A. Shah, P.S. Shrivastav, Natural biodegradable polymers based nanoformulations for drug delivery: A review, Int. J. Pharm. 561 (2019) 244- 264, https://doi.org/10.1016/j.ijpharm.2019.03.011.

[6] R.R.G. Soares, A.M. Azevedo, J.M. Van Alstine, M.R. Aires-Barros, Partitioning in aqueous two-phase systems: Analysis of strengths, weaknesses, opportunities and threats, Biotechnol. J. 10 (2015) 1158-1169, https://doi.org/10.1002/ biot.201400532.

[7] A.G. Teixeira, R. Agarwal, K.R. Ko, J. Grant-Burt, B.M. Leung, J.P. Frampton, Emerging Biotechnology Applications of Aqueous Two-Phase Systems, Adv. Healthc. Mater. 7 (2018) 1-19, https://doi.org/10.1002/adhm.201701036.

[8] M.A. Torres-Acosta, K. Mayolo-Deloisa, J. González-Valdez, M. Rito-Palomares, Aqueous Two-Phase Systems at Large Scale: Challenges and Opportunities, Biotechnol. J. 1800117 (2018) 1800117, https://doi.org/10.1002/ biot.201800117. 
[9] F. Ruiz-Ruiz, J. Benavides, O. Aguilar, M. Rito-Palomares, Aqueous two-phase affinity partitioning systems: Current applications and trends, J. Chromatogr. A. 1244 (2012) 1-13, https://doi.org/10.1016/j.chroma.2012.04.077.

[10] Y. Chao, H.C. Shum, Emerging aqueous two-phase systems: from fundamentals of interfaces to biomedical applications, Chem. Soc. Rev. 49 (2020) 114-142, https://doi.org/10.1039/C9CS00466A.

[11] R.J.H. Stenekes, O. Franssen, E.M.G. van Bommel, D.J.A. Crommelin, W.E. Hennink, The use of aqueous PEG/dextran phase separation for the preparation of dextran microspheres, Int. J. Pharm. 183 (1999) 29-32, https://doi.org/ 10.1016/S0378-5173(99)00038-1.

[12] S.D. Hann, K.J. Stebe, D. Lee, All-Aqueous Assemblies via Interfacial Complexation: Toward Artificial Cell and Microniche Development, Langmuir 33 (2017) 10107-10117, https://doi.org/10.1021/acs. langmuir.7b02237.

[13] F. Dumas, E. Roger, J. Rodriguez, L. Benyahia, J.-P. Benoit, Aqueous Two-Phase Systems: simple one-step process formulation and phase diagram for characterisation, Colloid Polym. Sci. 298 (2020) 1629-1636, https://doi.org/ 10.1007/s00396-020-04748-8.

[14] G. Balakrishnan, T. Nicolai, L. Benyahia, D. Durand, Particles trapped at the droplet interface in water-in-water emulsions, Langmuir 28 (2012) 5921- 5926, https://doi.org/10.1021/la204825f.

[15] E. Scholten, L.M.C. Sagis, E. van der Linden, Effect of bending rigidity and interfacial permeability on the dynamical behavior of water-in-water emulsions, J. Phys. Chem. B. 110 (2006) 3250-3256, https://doi.org/10.1021/ jp056528d.

[16] M.W. Edelman, E. Van Der Linden, R.H. Tromp, Phase separation of aqueous mixtures of poly(ethylene oxide) and dextran, Macromolecules 36 (2003) 7783-7790, https://doi.org/10.1021/ma0341622.

[17] D.M.A. Buzza, P.D.I. Fletcher, T.K. Georgiou, N. Ghasdian, Water-in-water emulsions based on incompatible polymers and stabilized by triblock copolymers-templated polymersomes, Langmuir 29 (2013) 14804-14814, https://doi.org/10.1021/la403356j.

[18] M. Destribats, S. Gineste, E. Laurichesse, H. Tanner, F. Leal-Calderon, V. Héroguez, V. Schmitt, Pickering emulsions: What are the main parameters determining the emulsion type and interfacial properties?, Langmuir 30 (2014) 9313-9326, https://doi.org/10.1021/la501299u.

[19] B.P. Binks, H. Shi, Phase Inversion of Silica Particle-Stabilized Water-in-Water Emulsions, Langmuir 35 (2019) 4046-4057, https://doi.org/10.1021/acs. langmuir.8b04151.

[20] K. Golemanov, S. Tcholakova, P.A. Kralchevsky, K.P. Ananthapadmanabhan, A. Lips, Latex-Particle-Stabilized Emulsions of Anti-Bancroft Type, Langmuir 22 (2006) 49684977, https://doi.org/10.1021/la0603875.

[21] N. Abbasi, M. Navi, S.S.H. Tsai, Microfluidic Generation of Particle-Stabilized Water-inWater Emulsions, Langmuir $34 \quad$ (2018) 213-218, https://doi.org/ 10.1021/acs.langmuir.7b03245.

[22] B.T. Nguyen, T. Nicolai, L. Benyahia, Stabilization of water-in-water emulsions by addition of protein particles, Langmuir 29 (2013) 10658-10664, https://doi. org/10.1021/la402131e.

[23] A.A.K. Das, B.W. Filby, D.A. Geddes, D. Legrande, V.N. Paunov, High throughput fabrication of cell spheroids by templating water-in-water Pickering emulsions, Mater. Horizons 4 (2017) 2017, https://doi.org/10.1039/ c7mh00677b.

[24] H. Liang, B. Zhou, J. Li, Y. He, Y. Pei, B. Li, Engineering functional alginate beads for encapsulation of Pickering emulsions stabilized by colloidal particles, RSC Adv. 6 (2016) 101267-101276, https://doi.org/10.1039/C6RA21755A.

[25] I. Minkov, T. Ivanova, I. Panaiotov, J. Proust, P. Saulnier, Reorganization of lipid nanocapsules at air-water interface: I. Kinetics of surface film formation, Colloids Surfaces B Biointerfaces 45 (2005) 14-23, https://doi.org/10.1016/ j.colsurfb.2005.03.009.

[26] I. Minkov, T. Ivanova, I. Panaiotov, J. Proust, P. Saulnier, Reorganization of lipid nanocapsules at air-water interface: Part 2. Properties of the formed surface film, Colloids Surf. B. Biointerfaces. 44 (2005) 197-203, https://doi.org/ 10.1016/j.colsurfb.2005.07.001.

[27] N. Matougui, L. Boge, A.-C. Groo, A. Umerska, L. Ringstad, H. Bysell, P. Saulnier, Lipidbased nanoformulations for peptide delivery, Int. J. Pharm. 502 (2016) 80-97, https://doi.org/10.1016/j.ijpharm.2016.02.019.

[28] B. Heurtault, P. Saulnier, B. Pech, J.E. Proust, J.P. Benoit, A novel phase inversion -based process for the preparation of lipid nanocarrier, Pharm. Res. 19 (2002) 875-880.

[29] K. Messaoudi, P. Saulnier, K. Boesen, J.-P. Benoit, F. Lagarce, Anti-epidermal growth factor receptor siRNA carried by chitosan-transacylated lipid nanocapsules increases sensitivity of glioblastoma cells to temozolomide, Int. J. Nanomed. 9 (2014) 1479-1490, https://doi.org/10.2147/IJN.S59134.

[30] A. Kaul, The phase diagram, Methods Biotechnol. 11 (2000) 11-21, https://doi. org/10.1385/1-59259-028-4:11.

[31] J.-P. Douliez, N. Martin, T. Beneyton, J.-C. Eloi, J.-P. Chapel, L. Navailles, J.-C. Baret, S. Mann, L. Béven, Preparation of Swellable Hydrogel-Containing Colloidosomes from Aqueous Two-Phase Pickering Emulsion Droplets, Angew. Chemie Int. Ed. 57 (2018) 7780-7784, https://doi.org/10.1002/ anie.201802929.

[32] S.U. Pickering, CXCVI-Emulsions, J. Chem. Soc., Trans. 91 (1907) 2001-2021, https://doi.org/10.1039/CT9079102001.

[33] S. Hirsjärvi, G. Bastiat, P. Saulnier, J.P. Benoît, Evaluation of surface deformability of lipid nanocapsules by drop tensiometer technique, and its experimental assessment by dialysis and tangential flow filtration, Int. J. Pharm. 434 (2012) 460-467, https://doi.org/10.1016/j.ijpharm.2012.06.019.

[34] S. Hirsjärvi, S. Dufort, G. Bastiat, P. Saulnier, C. Passirani, J.-L. Coll, J.-P. Benoît, Surface modification of lipid nanocapsules with polysaccharides: from physicochemical characteristics to in vivo aspects, Acta Biomater. 9 (2013) 6686-6693, https://doi.org/10.1016/j.actbio.2013.01.038.
[35] L. Alison, P.A. Rühs, E. Tervoort, A. Teleki, M. Zanini, L. Isa, A.R. Studart, Pickering and Network Stabilization of Biocompatible Emulsions Using Chitosan-Modified Silica Nanoparticles, $\quad$ Langmuir. $\quad 32 \quad$ (2016) 13446-13457, https://doi.org/10.1021/acs.langmuir.6b03439.

[36] M.M. Eissa, R.M. El-Moslemany, A.A. Ramadan, E.I. Amer, M.Z. El-Azzouni, L.K. ElKhordagui, Miltefosine Lipid Nanocapsules for Single Dose Oral Treatment of Schistosomiasis Mansoni: A Preclinical Study, PLoS One 10 (2015), https:// doi.org/10.1371/journal.pone.0141788 e0141788.

[37] P. Resnier, P. LeQuinio, N. Lautram, E. André, C. Gaillard, G. Bastiat, J.-P. Benoit, C. Passirani, Efficient in vitro gene therapy with PEG siRNA lipid nanocapsules for passive targeting strategy in melanoma, Biotechnol. J. 9 (2014) 1389-1401, https://doi.org/10.1002/biot.201400162.

[38] N. Skandrani, A. Barras, D. Legrand, T. Gharbi, H. Boulahdour, R. Boukherroub, Lipid nanocapsules functionalized with polyethyleneimine for plasmid DNA and drug codelivery and cell imaging, Nanoscale 6 (2014) 7379, https://doi. org/10.1039/c4nr01110d.

[39] S.D. Hann, K.J. Stebe, D. Lee, AWE-somes: All Water Emulsion Bodies with Permeable Shells and Selective Compartments, ACS Appl. Mater. Interfaces 9 (2017) 25023-25028, https://doi.org/10.1021/acsami.7b05800.

[40] P. Liu, X. Zhao, Facile preparation of well-defined near-monodisperse chitosan/sodium alginate polyelectrolyte complex nanoparticles (CS/SAL NPs) via ionotropic gelification: A suitable technique for drug delivery systems, Biotechnol. J. 8 (2013) 847-854, https://doi.org/10.1002/ biot.201300093.

[41] J.Y. Seo, B. Lee, T.W. Kang, J.H. Noh, M.J. Kim, Y.B. Ji, H.J. Ju, B.H. Min, M.S. Kim, Electrostatically Interactive Injectable Hydrogels for Drug Delivery, Tissue Eng. Regen. Med. 15 (2018) 513-520, https://doi.org/10.1007/s13770-018-0146-6.

[42] Q. Ma, Y. Song, J.W. Kim, H.S. Choi, H.C. Shum, Affinity Partitioning-Induced SelfAssembly in Aqueous Two-Phase Systems: Templating for Polyelectrolyte Microcapsules, ACS Macro Lett. 5 (2016) 666-670, https://doi.org/10.1021/ acsmacrolett.6b00228.

[43] J.D. Schulz, M.A. Gauthier, J.-C. Leroux, Improving oral drug bioavailability with polycations?, Eur. J. Pharm. Biopharm. 97 (2015) 427-437, https://doi. org/10.1016/j.ejpb.2015.04.025. 